\title{
Overview of Modern and Traditional Techniques of Permeation Enhancement for Topical Drug Deliver
}

\section{Manan Patel ${ }^{1,2 *}$, Rigved Nagarkar ${ }^{1}$, Srushti Sodha ${ }^{1}$, Harshil M Parikh ${ }^{3}$ and Sowmya Kulal ${ }^{4}$}

${ }^{1}$ University of the Sciences in Philadelphia, PA, USA

${ }^{2}$ Biolink Life Sciences, NC, USA

${ }^{3}$ Teva Pharmaceutical Salt Lake City, USA

${ }^{4}$ Alcami, NC, USA

*Corresponding Author: Manan Patel, Department of Pharmaceutical Sciences, University of the Sciences in Philadelphia, USA.
Received: November 01, 2021

Published: December 09, 2021

(C) All rights are reserved by Manan Patel., et al.

\section{Abstract}

Although solid-oral dosage forms have the market and patient tolerance monopoly, the transdermal route is emerging as an attractive, non-invasive approach to deliver drugs topically and systemically. The skin is designed to prevent entry of foreign molecules, thereby presenting hurdles to drug permeation. Different penetration enhancers help circumvent this problem as they act at different sites of action and have different mechanisms of action. These include altering metabolic activity within the skin exerting an influence on the thermodynamic activity of the drug in its vehicle, changing metabolism rate inside the epidermis and/or influencing the thermodynamics action of the drug in its carrier. A variety of cutaneous permeation enhancers were studied and evaluated for skin permeation enhancement. The aim of this review article is to highlight various FDA approved pharmaceutical permeation enhancers and understand their mechanisms of action. A deeper dive into traditional and modern permeability enhancement techniques and understand their mechanisms which might help extrapolate the relevance of these technique/excipients for case-specific permeation enhancement.

Keywords: Skin; Topical; Delivery; Cutaneous; Penetration; Iontophoresis; Electroporation; Sonophoresis; Microfabrication Microneedles Technology; Needleless Injection; Thermophoresis; Magnetophoresis; Ceramide Analogs; Azone Analogs; Menthol Analogs; Transkarbams; Laser Radiations

\section{Introduction}

Topical medication application/administration has a lengthy history dating back to prehistoric times. Researchers in the ancient history may have discovered, administering an antidote/medication at the infected location, like inflammation, was frequently an appropriate method of curing. This method of drug administration had long been used in traditional medicine throughout the world. Topical drug delivery is widely used in modern health, particularly in over-the-counter preparations. The ability to successfully penetrate the molecule through the skin layer is critical to the effectiveness of topical administration. Outer most layer of the skin; stra- tum corneum's primary function is to provide protection against foreign particulates entering the body via skin. Ability of molecule's penetration through the stratum corneum and other skin layers determines the effectiveness of topically applied formulation [1].

To overcome the penetration challenges, one of the solutions is to use of penetration enhancers in the formulations. A class of penetration enhancers which reversibly alter the structure of the skin are used effectively in marketed products. Chemical enhancers like Dimethyl sulfoxide (DMSO) dissolve the lipids present in the cell bilayer thus increasing the permeability of drugs into the skin. Physical methods include application of low voltage electric 
current across the skin to increase permeability of the skin which helps diffusion of ionic drugs across the skin.

The skin is a multilayer structure with several layers. This is the most accessible organ in the body. The primary tasks include protecting important or critical inner organs from external stimuli, regulating temperature, controlling water outflow, and sensibility. The human skin on an average healthy person has a contact area approximately 2 sq $\mathrm{m}$ and gets roughly $1 / 3^{\text {rd }}$ of total blood circulation. The thickness of multilayer epidermal ranges based on cell number and size of cell layer, extending between $0.8 \mathrm{~mm}$ upon palms and soles around $0.6 \mathrm{~mm}$. They generate melanina, a yellowish - brown black pigmentation that adds to skin color and absorbs harmful UV radiation. The Langerhans cell develops from bone marrow and moves to the epidermal, which also makes up a tiny proportion of an epidermal cells. Dermis is around $0.3-0.8 \mathrm{~mm}$ in thickness of connective tissues that includes blood arteries, lymphatic system, and nerve. It is indeed the deepest and largest ( 90 percent) cutaneous layer, consisting largely of connective tissues and supporting the epidermal surface. The dermal-epidermal junction has been the border between epidermis and dermis layers. The epidermis and dermis are supported by the hypodermis, or subcutaneous fat cells. It functions like a fat storage place. The covering aids in temperature regulation, nutrition support, and mechanical protection. It connects major blood arteries and nerve to the surface, and may include sensory organs. The hypodermal layer is made up of flexible connective tissue and differs in thickness [2].

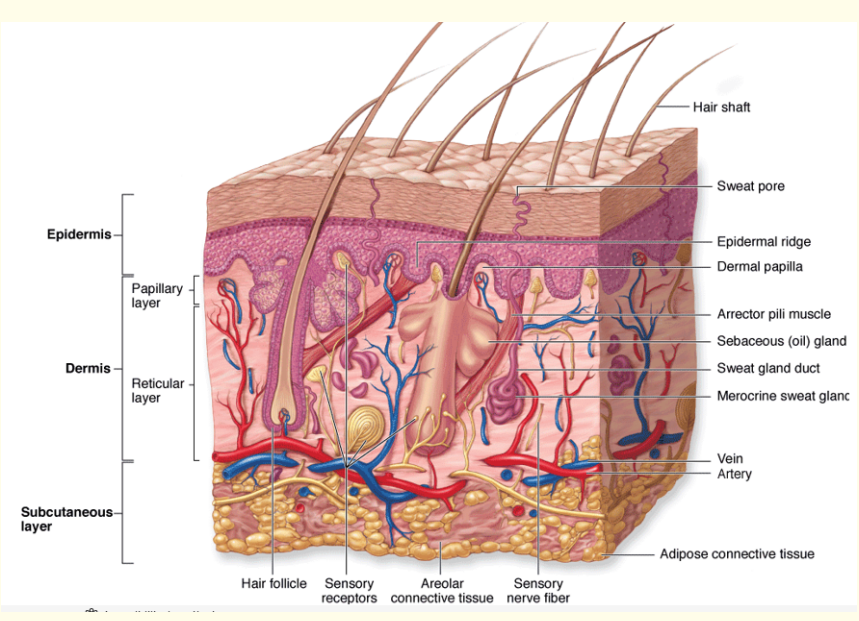

Figure 1: Schematic Diagram of Skin (N'Da, David. (2014). Molecules (Basel, Switzerland).
This review paper details a comprehensive summary on not only different types of penetration enhancers used but also discusses on the recent and advanced techniques used to increase permeability of drugs into the biological barrier.

\section{Principles}

Numerous substances have been tested as penetration enhancer in animal or human skin, only few have shown promising results. Some of the most desired features of penetration enhancer that work inside the skin are as follows [3]:

- These should not be poisonous, irritants, or allergens

- $\quad$ These must preferably work quickly, with consistent and stable action and period of effect

- These must not have any pharmacological action inside the body, these must not attach to receptor sites.

- The penetration enhancer must function unidirectionally, allowing medicinal substances penetrate while limiting the elimination of endogenous materials

- $\quad$ Once separated from epidermis, the barrier characteristics must recover quickly and completely

- The enhancers must be suitable for composition it in to a variety of topical treatments and therefore suitable both with ingredients and therapeutics

- $\quad$ These must be aesthetically appealing and have a natural skin 'feel'.

\section{Penetration enhancement techniques}

The numerous approaches discovered for improving penetration throughout the skin barrier are roughly categorized as follows.

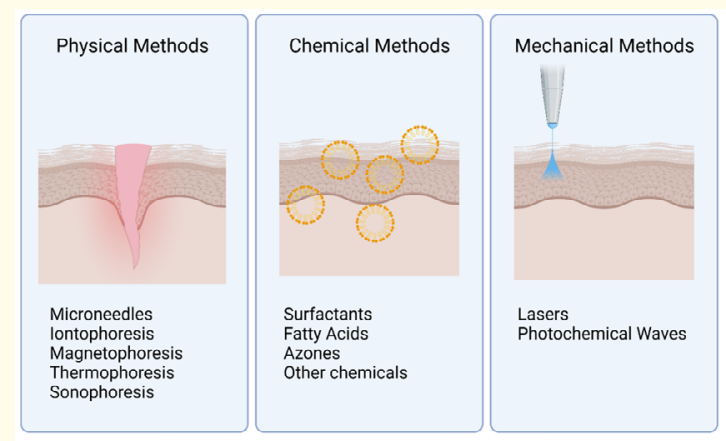

Figure 2: Methods of permeation enhancement: physical, chemical and mechanical. 
Physical techniques

Iontophoresis

The approach includes applying a low voltageelectric charge to skin to improve the penetration of topical application of a medicinal substance $[4,5]$. The methodology's increased drug penetration could be attributable with one or a blend of electro-perturbation, electro-repulsion, and electro-osmosis processes. Type of electrode, intensity of current, system $\mathrm{pH}$, competing ion action, and permeant form are all factors that influence the construction of an iontophoretic cutaneous delivery method [6]. The Phoresor ${ }^{\mathrm{TM}}$ device (Iomed Inc., Salt Lake City, UT) was the very first iontophoretic systems authorized by the FDA as a physical healthcare therapy device in the 1970s.Over decades, the usage of patient-friendly, accessible, and effective iontophoretic devices has been under active research in order to improve therapeutic efficacy. E-Trans and the Vyteris device are two examples of enhanced systems. The regulations upon current amount which may be utilized in people (now fixed at $0.5 \mathrm{~mA} / \mathrm{cm}^{2}$ ) as well as the permanent damage these currents may bring to skin barrier characteristics are among the drawbacks of iontophoretic devices. Furthermore, it has not considerably improved the transport of macromolecules larger than 7,000 Da [7].

\section{Electroporation}

Electroporationis the formation of hydrophilic pocketsinside the bilayer lipid membrane caused by the effect of small electrical signals ranging from 100 to $1000 \mathrm{~V} / \mathrm{cm}$. Charged particles have seen flux improvements to 10,000 folds. The effectiveness of movement is determined by electrical factors as well as drug physical and chemical properties. High - voltage power pulses are readily sustained in vivo; however muscular contractions are frequently produced. The electrodes and patches layout are critical in reducing the pain of electrical therapy in people [8]. Several investigations have shown that electroporation-mediated transport of polysaccharides, peptides, genes, oligonucleotides, is possible [9].The improvement is used for topical application of macromolecules with a molecular weight approximately $40 \mathrm{kDa}$ [10]. Even though DNA initiation has been the most commonly used system for electrophoresis, it was also used on cell cultures for insertion of enzymes, monoclonal antibody, and pathogens, but more lately, tissue electroporation had also started to also be investigated, with possible applications such as improved cancer chemotherapy, genetic modification, and tissue regeneration [11].

\section{Sonophoresis}

The technique of delivery of the drug into the skin by induced convection with use of ultrasound is called as Sonophoresis. Tang., et al. proposed a hypothesis for the epidermal transfer of aqueous permeants within context of ultrasound. Ultrasound modifies the epidermal permeable routes by two methods: (1) expanding the skin's pore size radius, or (2) creation of additional temporary pores [12]. According to recent research, it may enhance the capacity of size of protein of insulin to enter the epidermis by up to 5,000 times. The frequency of the ultrasonic wave is an essential consideration. Long term exposure at higher frequency, on the other hand, may impact the skin structure [13].

The hypothesized process for improvement in permeability is ascribed to development of gaseous spaces inside the lipid bilayer in response to ultrasonic treatment, leading in stratum corneum rupture. Therapy time, severity, and frequency have all been reported to stimulate skin absorption, the latter being most relevant [14]. While frequency ranging from $20 \mathrm{kHz}-16 \mathrm{MHz}$ have now been observed to improve skin penetration, frequency just at lower end (100 kHz) are thought to provide a more substantial influence on macromolecule distribution $[15,16]$.

\section{Microfabrication microneedles technology}

It makes use of micron-sized silicon needles. Microneedles of various sizes, shapes, and substances were created. After being inserted into epidermis, such microneedle grids provide channels for delivering drugs around the cell membrane [17]. Microneedles put in skin surface are found to be noninvasive. Microneedle drug delivery improves skin penetration of various nanocarriers as well as macro molecules [18].

The approach formed the basis for one of earliest inventions being submitted for a medication delivery system for cutaneous administration of drugs. According to the invention, the device consists of such a drug reservoir and a number of protrusions protruding from reservoirs. These 50-110 mm long microneedles can enter the cell membranes and epidermal to release the medication from reservoir. The reservoirs could include drug, a drug solution, gels, or solid materials, and various implementations of innovation also include employment of a wall to isolate the medication from epidermis and manage release of drug out of its reservoir [19]. 


\section{Needleless injection}

It is said to be a painless technique of delivering medicines to a surface. As a result, this approach eliminates the concerns about safety, discomfort, and anxiety that are connected through the use of hypodermic needles. The delivery is accomplished by propelling solid or liquid particle at light speed via the skin's outermost layer with an appropriate source of energy [20].

- Intra-Ject-is prefilled disposable device injector that is currently being developed. This is meant to employ a nitrogen driven device with an empty medication capsule.

- Implaject- inserts a small, potentially "Pioneer tip" through the surface before of the medication.

- Jet Syringes- are available with either an adjustable dosage "fill and shoot" ampoule or even a pre-filled ampoule in fixed dose purposes [21].

\section{Thermophoresis}

In human beings, a variety of homeostatic mechanisms keep the surface of the skin temperatures around $32^{\circ} \mathrm{C}$. First, the impact of increased temperature on skin penetration was documented. It had lately become a rise in research in utilizing thermoregulation to improve the distribution profile of topical medications. Earlier in vitro research $[22,23]$ have shown that each $7-8^{\circ} \mathrm{C}$ rise in epidermal temperature rise causes a 2-3-fold improvement in flux. Enhanced penetration after heating has already been ascribed to increased medication diffusivity in the liquid and even in the epidermis owing to enhanced lipid permeability [24].

\section{Magnetophoresis}

A magnetic field is used as an extrinsic driving factor to promote the passage of a diamagnetic fluid through the epidermis throughout this technique. Skin contact to a magnetic flux may potentially cause structural changes which lead to increased permeability. In vitro experiments revealed a magnetic-induced rise in benzoic acid flow, that increased with intensity of a strong electric field [25].

\section{Chemical techniques}

\section{Ceramide analogs}

Ceramides, fatty acid and cholesterol, or trace of cholesterol sulfate, make up the phospholipids in the stratum corneum [26,27]. These are known to be the core constituents accountable for stratum corneum barrier action. These constitute amphiphilic compounds with long hydrocarbon tail as well as an acylamide hydrophilic head [28,29]. According to Vavrova., et al. there may be structural similarities among ceramide and enhancer, allowing enhancers particles to position itself between hydrophobic tails of ceramide lipid bilayer and decrease the continuation of a phospholipid barrier [30]. A variety of ceramide metabolites with two types of polar head groups centered on glycine and L-serine had been produced in this investigation, and also the link here between characteristics of a polar head groups and enhancing potential was explored. The results showed whereas these molecules were ineffective in isopropyl myristate, these had substantial enhancing effects while employed in suspension. Ceramide derivatives with 6 distinct side chains (compounds 7e12) and 8 different polar group (compounds $7,13,15 \mathrm{e} 20$ ) have been prepared to further investigate the above hypothesis [31]. While derivatives of same side chain were examined, compounds 18 and 7 showed the greatest enhancing impact. The chemical products exhibited the shortest polar head group and the weakest H-bonding capability, confirming the prior hypothesis of lowering the size or H-bonding capacity of polar head groups of ceramide derivatives will lead to their enhancing activity.

\section{Azone analogs}

The very first chemical specifically developed as an epidermal permeability enhancer was azone (1-dodecylazacycloheptan2-one or laurocapram). That has been shown to improve the transdermal uptake of a broad range of medicines [32]. Azone's permeation-enhancing actions are most likely mediated by interactions with stratum corneum phospholipid domains. Azone's composition is split into two sections: the non- polar chain as well as the hydrophilic head (heterocycle and carbon on non-polar chain). The most prevalent structural alteration to a hydrophobic tail had been a decrease in side chain length.

The optimum hydro-carbon chain lengths for Azone derivatives have always been found that between 8 and 14 carbon atoms, which varied depending on the polar head groups. Because the activity of even an enhancer were thought to be reliant mostly on ratio of its lipophilic and hydrophilic characteristics, lengths of alkyl chains were necessary for optimum integration of an enhancer with varied polar heads. A research [33] looked at the influence of a heteroatom inside the polar head groups of Azone derivatives. The elimination of a nitrogen atom with in polar head groups of compounds 27 and 28 led to a significant reduction inside the promoting activity that compounds 29 and 30 exhibited activities. 
Menthol analogs

Obata., et al. discovered how an ether analog of menthol, O- ethylmenthol, showed good efficacy in vivo absorption of ketoprofen hydrogels via rabbit skin [34,35]. Depending on this discovery, a variety of mono- and di- substituted cyclohexane compounds containing an 0-ethyl groups were produced and tested for absorbing enhancing activities. The research also showed that irritant potency of such compounds corresponded well enough with boosting action, but both exhibited parabolically dependent upon substances' $\log$ P. As just an example, 1-0-ethyl-3-butylcyclohexanol has been demonstrated being a suitable and significant option which increased the absorption of 10 distinct type medicines while causing relatively little discomfort [36,37]. Zhao., et al. used menthol as a starting point and produced $\mathrm{O}$-acylmenthol compounds using a variety of saturated fats (C2-C18) and medical excipient acids [38]. In fatty acid esters, all had a good boosting impact also on transdermal absorption of ketoprofen, indomethacin, and isosorbide dinitrate, with compound 88 and 89 producing the largest enhancing action towards isosorbide dinitrate and indomethacin. Compounds having short acyl chains, like molecules 85 and 86, typically offered the highest boosting efficacy for ketoprofen.

\section{Transkarbams}

Because of its safety and well-known constituents, amino acid ester has often been produced as biodegradable penetration enhancers. Dolezal., et al. discovered how an acyclic Azone analogue, dodecyl-6-aminohexanoate (DDEAC), had significantly greater efficacy and less toxicity as that of Azone. This was discovered that enhancer's action did not interact with that of an amino ester molecule. The free amino group in DDEAC could interact with $\mathrm{CO} 2$ to produce additional 2-chain enhancer with such a carbamate salt creating its polar head group, known as Transkarbam 12 (T12), which has been proposed to be accountable for boosting action [39]. These analogs with ending ethyl or methyl branches also were synthesized in Part III, and its stimulating action was compared to those of its linear derivatives [40]. Each of these transkarbams showed a parabolic connection among side chain length and permeation boosting action. The research also indicates also that ammonium salts (ammonium chloride) produced during carbamate breakdown was a potent enhancer molecule, but it's not as efficient as equivalent Transkarbams.

\section{TXA analogs}

Tranexamic acid (TXA) has been shown to enhance skin barrier homeostasis and also overall skin disease. Following on T12 research, a variety of TXA compounds were created as penetration enhancers, with the expectation that they would be converted into TXA in subcutaneous tissue and so repair barrier function. TXA has a composition comparable to 6-aminohexanoic acid, which would be the polar group of T12. The sole distinction among TXA and transkarbams derivatives would be that TXA has a cyclohexane ring rather than aliphatic-chains [41]. TX12 had outstanding efficacy both in water and IPM donor vehicles that was nearly twice as high as that of Azone. But its action still was less in T12 due to the relative stiffness of a cyclohexane cycle as well as the energetic undesirable shift in conformation. FTIR was used to confirm the breakdown of a carbamate salts of TX12 in a somewhat acidic environment. HPLC and TLC were used to detect the enzymolysis of a relevant esterification reaction by porcine enzyme for quantitative and qualitative research, demonstrating the emission of TXA throughout the skin. Mostly as consequence of TXA's beneficial impact mostly on skin healing process, simpler and quicker skin healing is predicted.

\section{DDAK and DDAIP}

DDAIP (dodecyl 2-(dimethylamino) propanoate) had been a powerful and biodegradable booster with good topical boosting action against a variety of medications, particularly clonidine, indomethacin, and hydrocortisone, adefovir [42-44]. The ionizable dimethylamino polar end of DDAIP as well as the 5-carbon connecting chain of T12 were combined to form DDAK. The sole distinction among DDAIP and DDAK is in the connecting groups, with DDAK having long chain length of 5 carbon atoms and DDAIP having a branch connecting group. To examine the 2 compounds in depth, 4 model medicines were chosen to assess the action of an enhancer, namely hydrocortisone, theophylline, and adefovir, indomethacin. The findings showed that DDAK had considerably higher efficacy than DDAIP for hydrocortisone, theophylline, and adefovir, but DDAIP was marginally superior for indomethacin $[45,46]$. Following that, the impact of varied connecting chain lengths was investigated using compounds 175-179. The ideal length of the connecting chain was proposed to have an important role in increasing the action of DDAK as well as its analogs.

\section{Sulphoxides}

Dimethyl sulphoxide (DMSO) was one of the first and perhaps most extensively researched penetration enhancer. This is a strong aportic solvent that attaches to hydrogen instead of water. This is tasteless, colorless, and hydroscopic, and this is frequently employed also as "universal solvent" in various fields of clinical medi- 
cine. For reducing systemic inflammation, it was used topically. As just a penetration enhancer, it acts quickly--spillage of a substance over to skin may be felt inside the tongue within just seconds. The enhancer's action is concentration-dependent, and typically cosolvents comprising more than $60 \%$ DMSO are required for optimal enhancing effectiveness. This can, though, produce erythema of SC at a relatively high dose. Erythema, scales, contact urticaria, itching, and burning sensations are caused by the denaturation of certain skin proteins [47]. Studies have examined a comparable synthetically substance as just an accelerant since it is difficult to be used as a permeation enhancer. Dimethylformamide (DMF) and Dimethylacetamide (DMA) are both strong aportic solvent. A research that found a 12 -fold rise in caffeine flow penetrating over DMF-treated human tissue indicated also that stimulant caused permanent cellular injury. DMF causes irreversible damage to skin membrane, although that has been shown to increase the bioavailability of betamethasone17-benzoate during in vivo study [48]. DMSO can also remove phospholipids, allowing the keratin layer to permeate more easily by creating porous channels. The method of sulphoxide permeation enhancers is frequently employed to degrade protein and been demonstrated to alter the interstitial keratin shape from $\alpha$ to $ß$ sheet when applied to skin $[49,50]$.

\section{Pyrrolidones}

These were employed to improve permeability of a variety of compounds, namely aqueous soluble (5-flurouracil and mannitol) and lipid soluble (hydrocortisone and progesterone) permeants. When formed in a matrix-type patch, $\mathrm{N}$-methyl-2-pyrolidone has been used with little effectiveness as an enhancer with captopril [51].

\section{Fatty acids}

A broad spectrum of lengthy chain fatty acid, a most common of which would be oleic acid, have been shown to enhance percutaneous medication uptake. This is important to note that several enhancers, like azone, comprise unsaturated or saturated hydrocarbon side chains, as well as some structure-activity interactions have indeed been depicted from Aungst's rigorous work, which used a variety of acids, fatty acids, sulphoxides, alcohols, and surfactants as enhancers for naloxone [52,53]. A research was conducted upon release of triprolidone using different enhancers such as fatty acids (lauric acid, capric acid, and myristic acid), glycols (tetraethylene and diethylene glycol), anionic surfactants (polyoxyethylene-2-stearly ether and (polyoxyethylene-2-oleyl ether) [54].
Terpenoids and terpenes

Terpenoids and terpenes are molecules present in essential oil that contain only oxygen, carbon, and hydrogen atoms so are not aromatic. These has historically been utilized as both medications and flavoring compounds. The essential oil of Chenopodium, eucalyptus, and ylang-ylang were discovered to be excellent enhancers for 5-fluorouracil transversing skin during in vivo [55]. The penetration of 5-flurouracil into skin surface was studied using 12 sesquiterpene [56]. The uptake of 5-flurouracil was enhanced when cutaneous membrane was pretreated with sesquiterpene oil or solid sesquiterpenes saturated in dimethyl isosorbide. L-menthol has been shown to aid in vitro penetration of morphine hydrochloride via shaved rat skin [57], and also diffusing of imipramine hydrochloride across skin surface and hydrocortisone via shaved rat skin [58,59].

Urea

It facilitates transdermal penetration by hydrating the SC and forming aqueous diffusion pathways inside the barrier. Cyclic urea enhancers are harmless, biodegradable compounds composed of a hydrophilic parent component and an alkyl chain esters ring.As an outcome, the enhanced process might be the consequence from both aqueous action and the lipid disruption process.

\section{Glycols and alcohols}

Ethanol is now a popular solvent utilized in several epidermal formulations and is frequently the solvent of selection for patch usage. This is also often used as a cosolvent with liquid in in vitro permeation tests to ensure sink condition. Ethanol, like water, penetrates fast into skin surface, with such a steady-state flow of around $1 \mathrm{mg} \mathrm{cm}^{2} / \mathrm{h}$ [60]. Ethanol was used in vivo to increase the flow of estradiol, levonorgestrel, hydrocortisone, and 5-fluorouracil via rat skin [61] or estradiol via human tissue. By utilizing an ethanol water cosolvent vehicle, though, the ethanol augmentation impact appears to just be dosage dependent [62].

\section{Surfactants}

Surfactants, like several of the discussed earlier compounds (propylene glycol and ethanol), could be widely used in a variety of medicinal, cosmetic, and agricultural applications. Surfactants are often included to compositions to solubilize lipid soluble active compounds, but they also have the ability to solubilize lipids inside the skin barrier. Surfactants have been frequently characterized in regardless of the nature of the aqueous component since they are usually constituted of a lipid soluble aryl or alkyl fatty chain and a 
hydrophilic head ring. Non-ionic surfactants comprise nonoxynol, cationic surfactants comprise cetyltrimethyl ammonium bromide, sodium lauryl sulphate (SLS), and zwitter ionic surfactants comprise dodecyl betaine. SLS seems to be a strong irritant that increases trans-epidermal loss of water in healthy volunteers in vivo, and that both cation and anion surfactants expand the skin barrier and react with interstitial keratin. Nonionic surfactant is generally considered as safe [63].

\section{Mechanical techniques}

\section{Laser radiations}

Use of such lasers to dissolve the skin barrier by regulated ablation has been studied as a way of improving topical medication delivery. The procedure allows the rapid and controlled application of such a laser to a skin, resulting in the destruction of a cell membrane while causing little damage to the underneath epidermis.This approach of removing the stratum corneum has already been found to improve the administration of hydrophilic and lipophilic medicines. It was also observed that laser ablation of cell membrane improved interferon and hydrocortisone penetration. Mechanical waves are produced by either directly irradiation of substance in skin contact by such a laser beam or restricted irradiation caused by an interface existing upon top of a irradiated substance [63].

\section{Photomechemical waves}

These are just the intensity pulses generated by Q-switched laser ablation of a chemical substrate (polystyrene). In vivo, a particular photomechanical wave modifies the protective barrier of stratum corneum without altering the viability or morphology of the epidermal or dermal layer. These have the potential to make the cell membrane more porous to macromolecules through a temporary permeabilization action caused by the development of transitory pathways. The administration of waves causes neither discomfort or pain, as well as the SC's intestinal barrier usually improves. Too far, the biggest chemical known to also be transported via rat skin has a molecular weight of 40,000 Da. Many therapeutically significant proteins, like hematoprotein (48000 Da) and insulin (6000 Da), were suggested to be within and near to a distribution capacity range. The comparatively recent method, though, doesn't really appear to have yielded significant human clinical evidence [64].

\section{Penetration enhancer-containing vesicles (PEVs)}

Such vesicles transporters are made by combining a PE with the conventional liposome formulation. Various hydrophilic and lipophilic enhancers (e.g., labrasol, cineole, transcutol, alkylglucosides) were employed, and the resulting PEVs had all been investigated for cutaneous administration of minoxidil, diclofenac, and tretinoin $[65,66]$. When opposed to traditional liposomal formulations presently on the industry, they were capable of improving cutaneous distribution of investigated medicines. Their characteristics, however, differed but were significantly dependent to the enhancers utilized. Its deformability was highly varied, but only a small number of these exhibited elastic characteristics, too as a factor of an injected medication. However, the non-deformable PEVs have been able to easily carry medicines towards the epidermis, as well as the findings lead us to speculate on its probable mechanism of action. Transcutol- PEVs did, in fact, enter the epidermis whole via a combination process combining the enhancer (transcutol), the vesicles transporter, as well as the epidermal lipid of aintercorneocyte network [67].

\begin{tabular}{|c|c|c|c|}
\hline Drug & $\begin{array}{c}\text { Penetration } \\
\text { enhancer }\end{array}$ & Results & Reference \\
\hline Tretinoin & $\begin{array}{c}\text { (Transcutol }{ }^{\circledR} \mathrm{P}, \mathrm{Trc} \text { ), } \\
\text { decylpolyglucoside } \\
\text { (Oramix }{ }^{\circledR} \mathrm{NS} 10, \\
\text { OrNS10), } \\
\text { 2- (2-ethoxyethoxy) } \\
\text { ethanolcaprylocaproyl } \\
\text { macrogol } \\
\text { 8-glyceride } \\
\text { (Labrasol }{ }^{\circledR} \text {, Lab), and } \\
\text { propylene glycol (PG) }\end{array}$ & $\begin{array}{c}\text { Indeed, the } \\
\text { inclusion of an } \\
\text { aqueous PE } \\
\text { component } \\
\text { throughout the } \\
\text { composition of } \\
\text { P50 liposomes } \\
\text { is critical for } \\
\text { enhancing } \\
\text { cutaneous } \\
\text { distribution of } \\
\text { a lipid soluble } \\
\text { medication, ac- } \\
\text { cording to ex vivo } \\
\text { permeation and } \\
\text { penetration } \\
\text { studies. }\end{array}$ & [68] \\
\hline Minoxidil & 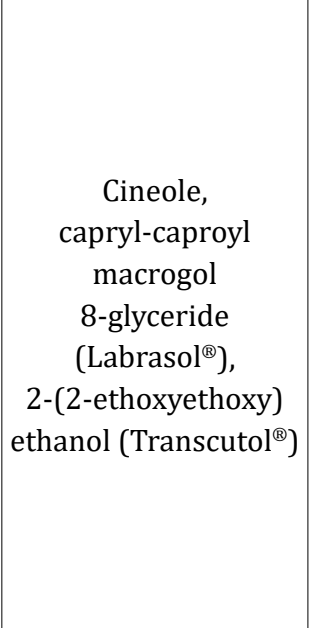 & $\begin{array}{c}\text { skin } \\
\text { penetration in } \\
\text { vitro results } \\
\text { revealed that } \\
\text { PEVs are capable } \\
\text { of providing } \\
\text { a significant } \\
\text { improvement } \\
\text { in minoxidil } \\
\text { accumulation } \\
\text { throughout } \\
\text { the skin when } \\
\text { compared to } \\
\text { traditional } \\
\text { liposomes. }\end{array}$ & [69] \\
\hline
\end{tabular}




\begin{tabular}{|c|c|c|c|}
\hline Quercetin & $\begin{array}{c}\text { Propylene glycol, } \\
\text { Transcutol }^{\circledR} \mathrm{P}, \\
\text { Labrasol }^{\circledR} \text { and } \\
\text { polyethylene glycol } \\
400\end{array}$ & \begin{tabular}{|} 
PEs' capacity to \\
boost \\
solubility of \\
drugs in vesicles \\
distribution, but \\
most \\
importantly, to \\
act \\
synergistically \\
with lipids as \\
penetration \\
enhancer,
\end{tabular} & [70] \\
\hline Sertaconazole & $\begin{array}{l}\text { Thioglycolic acid, } \\
\text { N-acetyl-L-cysteine, } \\
\text { thiourea, ethanol }\end{array}$ & $\begin{array}{c}\text { The chosen } \\
\text { composition had } \\
\text { a spherical shape } \\
\text { and a large } \\
\text { storage } \\
\text { durability. It } \\
\text { demonstrated } \\
\text { 1.4-fold greater } \\
\text { hydration and } \\
\text { medication } \\
\text { absorption } \\
\text { improvement } \\
\text { in nail clippings } \\
\text { as relative to a } \\
\text { conventionally } \\
\text { cream. }\end{array}$ & [71] \\
\hline Baicalin & Sorbitol & \begin{tabular}{|} 
Thein vitro skin \\
delivery \\
demonstrated \\
an enhanced \\
capacity of \\
sorbitol-PEVs \\
to promote \\
flavonoid \\
accumulation \\
throughout \\
the skin
\end{tabular} & [72] \\
\hline
\end{tabular}

Table 1: Few examples of PEVs.

\section{Future prospects}

Because of function and nature of an application site, effective topical medication administration necessitates several factors. It is important to remember that perhaps the skin's primary roles are protection and containment. But new medicinal products are be- ing created as a result of ongoing research into the construction, functioning, and physiochemical properties of human skin. Over several decade, substantial scientific interest has been focused to a hunt for optimal penetration enhancers. While numerous powerful enhancers have been found, their enhancing effects are frequently accompanied with toxicity, restricting their therapeutic use. In latest years, the application of a variety of biophysical methods has assisted in the knowledge about the nature of skin barrier and how substances interface with it and impact it. A greater knowledge of enhancer interactions with skin barrier, as well as the creation of structure activity relations, will assist in the design of enhancers having optimal properties and low toxicity.

\section{Conclusion}

Permeation through the skin enhancement technology has become an important growing concept which will considerably increase the scope of medicines appropriate for topical drug delivery systems, resulting in skin being among the primary routes of administration within the next decade. Through use of several forms of enhancers in the augmentation of drug absorption via epidermis has been demonstrated in research on the topic. This various penetration augmentation strategies seem encouraging. The emphasis must be on irritation on the skin, with the goal of choosing penetration enhancers that have optimal enhancing effects while causing the least number of allergic reactions.

\section{Bibliography}

1. Nagarkar R., et al. "Novel Emulsion base for vaginal yeast infection with half drug concentration". Inidan Journal of Pharmaceutical and Biological Research 8.3 (2020): 01-11.

2. Mishra A., et al. "Topical antibiotics and Semisolid Dosage Forms". International Journal of Pharmaceutical Erudition 4.3 (2014): 33-54.

3. Williams AC and Barry BW. "Penetration enhancers ??". Advanced Drug Delivery Reviews 64 (2012): 128-137.

4. Wang Y., et al. "Iontophoresis of hydrocortisone across hairless mouse skin: Investigation of skin alteration". Journal of Pharmaceutical Sciences 82.11 (1993): 1140-1144.

5. Weaver JC., et al. "Theory of electrical creation of aqueous pathways across skin transport barriers". Advanced Drug Delivery Reviews 35.1 (1999): 21-39. 
6. Guy RH., et al. "Iontophoresis: Electrorepulsion and electroosmosis". Journal of Controlled Release 641-3 (2000): 129-132.

7. Kanikkannan N. "Iontophoresis-based transdermal delivery systems". BioDrugs 16.5 (2002): 339-347.

8. Denet AR., et al. "Skin electroporation for transdermal and topical delivery". Advanced Drug Delivery Reviews 56.5 (2004): 659-674.

9. Banga AK and Prausnitz MR. "Assessing the potential of skin electroporation for the delivery of protein- and gene-based drugs". Trends in Biotechnology 16.10 (1998): 408-412.

10. Lombry C., et al. "Transdermal delivery of macromolecules using skin electroporation". Pharmaceutical Research 17.1 (2000): 32-37.

11. Zhao YL., et al. "Induction of cytotoxic T-lymphocytes by electroporation-enhanced needle-free skin immunization". Vaccine 24.9 (2006): 1282-1290.

12. Tang H., et al. "Theoretical description of transdermal transport of hydrophilic permeants: Application to low-frequency sonophoresis". Journal of Pharmaceutical Sciences 98.10 (2009): 3878.

13. Bommannan D., et al. "Sonophoresis. II. Examination of the Mechanism (s) of Ultrasound-Enhanced Transdermal Drug Delivery". Pharmaceutical Research: An Official Journal of the American Association of Pharmaceutical Scientists 6 (1992): 1043-1047.

14. Mitragotri S and Kost J. "Low-frequency sonophoresis: A review”. Advanced Drug Delivery Reviews 56.5 (2004): 589-601.

15. Mitragotri S., et al. "Ultrasound-mediated transdermal protein delivery". Science 269.5225 (1995): 850-853.

16. Liu H., et al. "Investigation into the potential of low-frequency ultrasound facilitated topical delivery of Cyclosporin A". International Journal of Pharmaceutical Sciences 326.1-2 (2006): 32-38.

17. Nagarkar R., et al. "A review of recent advances in microneedle technology for transdermal drug delivery". Journal of drug delviery science and technology (2020): 101923.

18. Prausnitz MR. "Microneedles for transdermal drug delivery". Advanced Drug Delivery Reviews 56.5 (2004): 581-587.

19. Yuzhakov V V. "Microneedle array, patch, and applicator for transdermal drug delivery - US 7658728 B2". 2.12 (2010): 1-16.
20. Mitragotri S. "Current status and future prospects". 5 (2006): 543-548.

21. Bremseth DL and Pass F. "Delivery of insulin by jet injection: Recent observations". Diabetes Technology and Therapeutics 3.2 (2001): 225-232.

22. Clarys P., et al. "In vitro percutaneous penetration through hairless rat skin: Influence of temperature, vehicle and penetration enhancers". European Journal of Pharmaceutics and Biopharmaceutics 46.3 (1998): 279-283.

23. Akomeah F., et al. "Effect of heat on the percutaneous absorption and skin retention of three model penetrants". European Journal of Pharmaceutical Sciences 21.2-3 (2004): 337-345.

24. Zweier. "Effects of temperature, surfactants and skin location". Bone 23.1 (2014): 1-7.

25. Steven M Singer., et al. "Magnetophoresis for enhancing transdermal drug delivery". Physiology and Behavior 176.3 (2019): 139-148.

26. Narishetty STK and Panchagnula R. "Effect of L-menthol and 1,8-cineole on phase behavior and molecular organization of SC lipids and skin permeation of zidovudine". Journal of Controlled Release 102.1 (2005): 59-70.

27. Wertz PW and Van Den Bergh B. "The physical, chemical and functional properties of lipids in the skin and other biological barriers". Chemistry and Physics of Lipids 91.2 (1998): 85-96.

28. Infrared spectroscopic study of lipid interaction in stratum corneum treated with transdermal absorption enhancers.

29. Hatta I., et al. "Novel method to observe subtle structural modulation of stratum corneum on applying chemical agents". Chemistry and Physics of Lipids Internet 163.4-5 (2010): 381389.

30. Vávrová K., et al. "L-serine and glycine based ceramide analogues as transdermal permeation enhancers: Polar head size and hydrogen bonding". Bioorganic and Medicinal Chemistry Lett 13.14 (2003): 2351-2353.

31. Vávrová K., et al. "Synthetic ceramide analogues as skin permeation enhancers: Structure-Activity relationships". Bioorganic and Medicinal Chemistry 11.24 (2003): 5381-90.

32. Ahad A., et al. "Chemical penetration enhancers: A patent review". Expert Opinion on Therapeutic Patents 19.7 (2009): 969-988. 
33. Michniak BB., et al. "In vitro evaluation of atone analogs as dermal penetration enhancers". International Journal of Pharmaceutical Sciences 161.2 (1998): 169-178.

34. Barratt G. "Promoting effect of O-ethylmenthol". International Journal of Pharmaceutical Sciences 454.2 (2013): 597-598.

35. Takahara J., et al. "Multi-objective simultaneous optimization based on artificial neural network in a ketoprofen hydrogel formula containing O-ethylmenthol as a percutaneous absorption enhancer". International Journal of Pharmaceutical Sciences 158.2 (1997): 203-210.

36. Takayama K., et al. "Formula optimization based on artificial neural networks in transdermal drug delivery". Journal of Controlled Release 62.1-2 (1999): 161-170.

37. Li CJ., et al. "Effect of 1-0-ethyl-3-butylcyclohexanol on the skin permeation of drugs with different physicochemical characteristics". International Journal of Pharmaceutical Sciences 2591-2 (2003): 193-198.

38. Zhao L., et al. "Transdermal delivery of penetrants with differing lipophilicities using 0 -acylmenthol derivatives as penetration enhancers". European Journal of Pharmaceutics and Biopharmaceutics 69.1 (2008): 199-213.

39. Hrabálek A., et al. "Synthesis and enhancing effect of Transkarbam 12 on the transdermal delivery of theophylline, clotrimazole, flobufen, and griseofulvin". Pharmaceutical Research 23.5 (2006): 912-919.

40. Klimentová J., et al. "Transkarbams with terminal branching as transdermal permeation enhancers". Bioorganic and Medicinal Chemistry Lett 18.5 (2008): 1712-1715.

41. Vávrová K., et al. "Biodegradable derivatives of tranexamic acid as transdermal permeation enhancers". Journal of Controlled Release 104.1 (2005): 41-49.

42. Novotný J., et al. "Dimethylamino acid esters as biodegradable and reversible transdermal permeation enhancers: Effects of linking chain length, chirality and polyfluorination". Pharmaceutical Research 26.4 (2009): 811-821.

43. Barratt G. "Interaction of indomethacin". International Journal of Pharmaceutical Sciences 454.2 (2013): 597-8.

44. Fujii M., et al. "Enhancement of skin permeation of miconazole by phospholipid and dodecyl 2- (N,N-dimethyl amino) propionate (DDAIP)". International Journal of Pharmaceutical Sciences 234.1-2 (2002): 121-128.
45. Vávrová K., et al. "Transdermal and dermal delivery of adefovir: Effects of $\mathrm{pH}$ and permeation enhancers". European Journal of Pharmaceutics and Biopharmaceutics 69.2 (2008): 597604.

46. Vávrová K., et al. "Permeation enhancer dodecyl 6- (dimethylamino)hexanoate increases transdermal and topical delivery of adefovir: Influence of pH, ion-pairing and skin species". European Journal of Pharmaceutics and Biopharmaceutics 70.3 (2008): 901-907.

47. Kligman AM. "Topical Pharmacology and Toxicology of Dimethyl Sulfoxide-Part 1". The Journal of the American Medical Association 193.10 (1965): 796-804.

48. Bennett SL., et al. "Optimization of bioavailability of topical steroids: non-occluded penetration enhancers under thermodynamic control". Journal of Pharmacy and Pharmacology 37.5 (1985): 298-304.

49. Oertel RP. "Protein conformational changes induced in human stratum corneum by organic sulfoxides: An infrared spectroscopic investigation". Biopolymers 16.10 (1977) : 2329-2345.

50. Anigbogu ANC., et al. "Fourier transform raman spectroscopy of interactions between the penetration enhancer dimethyl sulfoxide and human stratum corneum". International Journal of Pharmaceutical Sciences 125.2 (1995): 265-282.

51. Park ES., et al. "Effects of adhesives and permeation enhancers on the skin permeation of captopril". Drug Development and Industrial Pharmacy 27.9 (2001): 975-980.

52. Aungst BJ., et al. "Enhancement of naloxone penetration through human skin in vitro using fatty acids, fatty alcohols, surfactants, sulfoxides and amides". International Journal of Pharmaceutical Sciences 33.1-3 (1986): 225-234.

53. Aungst BJ. "Structure/Effect Studies of Fatty Acid Isomers as Skin Penetration Enhancers and Skin Irritants. Vol. 6, Pharmaceutical Research: An Official Journal of the American Association of Pharmaceutical Scientists". (1989): 244-247.

54. Shin SC and Lee HJ. "Controlled release of triprolidine using ethylene-vinyl acetate membrane and matrix systems". European Journal of Pharmaceutics and Biopharmaceutics 54.2 (2002): 201-206.

55. Herman A and Herman AP. "Essential oils and their constituents as skin penetration enhancer for transdermal drug delivery: A review". Journal of Pharmacy and Pharmacology 67.4 (2015): 473-485. 
56. CORNWELL PA and BARRY BW. "Sesquiterpene Components of Volatile Oils as Skin Penetration Enhancers for the Hydrophilic Permeant 5-Fluorouracil". Journal of Pharmacy and Pharmacology 46.4 (1994): 261-269.

57. Morimoto Y., et al. "In vitro skin permeation of morphine hydrochloride during the finite application of penetrationenhancing system containing water, ethanol and l-menthol". Biological and Pharmaceutical Bulletin 25.1 (2002): 134-136.

58. Jain AK., et al. "Transdermal drug delivery of imipramine hydrochloride. I. Effect of terpenes". Journal of Controlled Release 79.1-3 (2002): 93-101.

59. El-Kattan AF., et al. "The effect of terpene enhancer lipophilicity on the percutaneous permeation of hydrocortisone formulated in HPMC gel systems". International Journal of Pharmaceutical Sciences 198.2 (2000): 179-189.

60. Berner B., et al. "Ethanol: Water mutually enhanced transdermal therapeutic system II: Skin permeation of ethanol and nitroglycerin". Journal of Pharmaceutical Sciences 78.5 (1989): 402-407.

61. Friend D., et al. "Transdermal delivery of levonorgestrel I: Alkanols as permeation enhancers in vitro". Journal of Controlled Release 7.3 (1989): 243-50.

62. Pershing LK., et al. "Mechanism of Ethanol-Enhanced Estradiol Permeation Across Human Skin in Vivo. Vol. 7, Pharmaceutical Research: An Official Journal of the American Association of Pharmaceutical Scientists". (1990): 170-175.

63. Mathur V., et al. "Physical and chemical penetration enhancers in transdermal drug delivery system". (2010): 173-183.

64. Patel MN., et al. "Skin Penetration Enhancement Techniques Physical Approaches". International Journal of Pharmacy and Pharmaceutical Sciences 1.2 (2010): 62-72.

65. Mura S., et al. "Transcutol containing vesicles for topical delivery of minoxidil”. Journal of Drug Targeting 19.3 (2011): 189196.

66. Caddeo C., et al. "Inhibition of skin inflammation in mice by diclofenac in vesicular carriers: Liposomes, ethosomes and PEVs". International Journal of Pharmaceutical Sciences Internet 443.1-2 (2013): 128-36.

67. Manconi M., et al. "Ex vivo skin delivery of diclofenac by transcutol containing liposomes and suggested mechanism of vesicle-skin interaction". European Journal of Pharmaceutics and Biopharmaceutics 78.1 (2011): 27-35.
68. Manconi M., et al. "Penetration enhancer containing vesicles as carriers for dermal delivery of tretinoin". 412 (2011): 37-46.

69. Mura S., et al. "Penetration enhancer-containing vesicles ( PEVs ) as carriers for cutaneous delivery of minoxidil”. 380 (2009): 72-79.

70. Chessa M., et al. "Effect of Penetration Enhancer Containing Vesicles on the Percutaneous Delivery of Quercetin through New Born Pig Skin". (2011): 497-509.

71. Bseiso EA., et al. "Novel nail penetration enhancer containing vesicles "nPEVs" for treatment of onychomycosis". Drug Delivery 23.8 (2016): 2813-2819.

72. Manca ML., et al. "Sorbitol-penetration enhancer containing vesicles loaded with baicalin for the protection and regeneration of skin injured by oxidative stress and UV radiation". International Journal of Pharmaceutical Sciences Internet 555 (2019): 175-83.

\section{Assets from publication with us}

- Prompt Acknowledgement after receiving the article

- Thorough Double blinded peer review

- Rapid Publication

- Issue of Publication Certificate

- High visibility of your Published work

Website: www.actascientific.com/

Submit Article: www.actascientific.com/submission.php Email us: editor@actascientific.com

Contact us: +919182824667 